\title{
PRESENTE Y FUTURO DE LA UROLOGÍA PEDIÁTRICA
}

\author{
C. MÍGUELEZ LAGO*, J.Mª GARAT BARREDO**, P.D.E. MOURIQUAND*** \\ *Servicio de Urología Pediátrica. Hospital Materno Infantil del Complejo Hospitalario Universitario Carlos \\ Haya. Málaga. **Servicio de Urología Pediátrica Instituto de Urología, Nefrología y Andrología. \\ Fundación Puigvert. Barcelona. ${ }^{* * *}$ Urología Pediátrica. Hôspital Debrousse. Lyon. France.
}

Actas Urol Esp. 28 (2): 84-88, 2004

\section{Situación actual de la Urología Pediátrica en España}

Hay dos formas de enfocar la Urología Pediátrica (UP):

1. Basados en el paciente (parece la más real): es el estudio y tratamiento de la patología urológica congénita o adquirida desde la vida fetal al final de la edad pediátrica.

2. Basada en el especialista (parece la más profesional), es lo mismo que la anterior, pero realizada en unidades de dedicación específica y exclusiva a UP.

$\mathrm{Si}$ nos atenemos al concepto de paciente pediátrico con patología urológica, en España la tratan diversos especialistas: urólogos y cirujanos pediátricos principalmente, pero también ginecólogos (urología fetal), nefrólogos pediátricos (reflujos, litiasis, hematurias, etc.), pediatras (infecciones urinarias, reflujos...), cirujanos generales (hidroceles, maldescenso testicular...), cirujanos plásticos (hipospadias, genitales ambiguos...), endocrinólogos (criptorquidia...), por solo poner algunos ejemplos de la dispersión de especialidades que tocan aspectos, más o menos parciales o generales, de la UP.

Actualmente en España, no hay nada que regule y evite esta dispersión aberrante. Cada comunidad autónoma, cada provincia, cada ciudad y cada hospital funcionan diferente. No hay criterios objetivos buscando la excelencia asistencial para los niños con patología urológica. En muchas ocasiones esta patología ha sido causa de discusiones y enfrentamientos crónicos que conducen a la falta de colaboración y de diálogo entre diferentes servicios, con un gran perjuicio para el niño pero también para los especialistas enfrentados.
No podemos, por ahora, conocer cuántos niños son tratados en estas circunstancias en España.

Lo que sí podemos conocer es el ámbito de las Unidades de Urología Pediátrica Españolas. Lo primero que llama la atención es que la gran mayoría se iniciaron sin ninguna planificación de la Administración Pública ni de una política sanitaria (número de habitantes, geografia, natalidad, etc.) "Se iniciaron porque alguien se interesó, se formó en UP, quiso y... le dejaron dedicarse a ello". Parece mentira pero así se empezó. La necesidad crea el órgano.

Hoy existen 18 Unidades de Urología Pediátrica con una dedicación casi total o total, de su trabajo diario a UP.

Hemos realizado en el año 2001 una encuesta a estas 18 Unidades, obteniendo unos datos muy interesantes y útiles para analizar y reflexionar sobre ello:

\section{Dedicación a la UP}

Quince Unidades refieren una dedicación al $100 \%$, y tres al $75 \%$.

\section{Dependencia administrativa}

Cirugía Pediátrica 12, Urología 3, y tres más se declaran servicios independientes, aunque están dentro de departamentos de Cirugía Pediátrica.

Número de especialistas

En total 47, media 2,6 (R 2-5).

\section{Especialidad de origen}

De Urología 16 (34\%), de Cirugía Pediátrica 31 (66\%). 
Actividad asistencial en el año 2000

Por sólo referirnos a los aspectos más representativos, citaremos:

- Consulta externa. Número total de días al mes: 197, media 11 días al mes. Número total de visitas en consulta: 37.255 pacientes, media 2.069 (R 980-4.243).

- Actividad quirúrgica. Número total de días al mes: 147, media 8 días al mes. Número total de operaciones con anestesia general: 4.948, media 274 (R 100-445).

\section{Guardias específicas de Urología Pediátrica}

Sólo 5 Unidades tienen este tipo de guardias: son las que hacen trasplantes renales. Sus guardias son realmente guardias para trasplante.

Docencia de residentes y becarios

- Reciben residentes de Urología: 16 Unidades (89\%), pero, sólo en 7 de ellas, pasan todos los residentes de Urología; en las 9 restantes, sólo pasan algunos de los residentes.

- Reciben residentes de Cirugía Pediátrica: 14 Unidades (78\%), pero en las 14, pasan todos los residentes de Cirugía Pediátrica.

- Reciben becarios nacionales o extranjeros: 10 Unidades (55\%), de ellas, 6 reciben más de un becario al año.

\section{CONSIDERACIONES}

El análisis de la situación actual se debe considerar desde dos aspectos diferentes:

\section{I- FACTORES POSITIVOS: FORTALEZAS}

- Experiencia. La edad media de los urólogos pediátricos españoles en activo es de 48 años ( $R$ 32-65), con una dedicación específica a la UP de 17 años de media. De las 18 Unidades de UP, 14 tienen más de veinte de existencia.

- Calidad. Es difícil escribir uno mismo acerca de la calidad de su trabajo, pero, siendo objetivos, podemos referirnos a un buen nivel, gozando de prestigio internacional bastantes de nuestras Unidades de UP. Como consecuencia, $10 \mathrm{de}$ ellas reciben con frecuencia becarios de otros países, procedentes de la Urología y de la Cirugía Pediátrica.

- Buena relación y entendimiento, en general, entre los UP, lo cual, facilita consensuar actitudes ante inquietudes comunes.
- Procedencia de ambas "especialidades madres”. Urología y Cirugía Pediátrica. Esto hace que el colectivo tenga una formación más completa y complementaria, junto a una facilidad para coordinar actividades, esfuerzos y proyectos.

\section{II- FACTORES NEGATIVOS: DEBILIDADES}

- Falta de planificación. No existe un plan de necesidades de UP de la población española. No se ha hecho un estudio actualizado y real (no teórico) de la prevalencia de patologías subsidiarias de valoración y tratamiento por UP. "Sabemos cuántos somos pero no cuántos deberíamos ser".

- Centralización. Como consecuencia de lo anterior, nos encontramos con cinco Unidades independientes en la ciudad de Madrid y con tres en Barcelona. Frente a Comunidades Autónomas donde no hay ninguna.

- Falta de regulación. En los 18 hospitales con UP, en general, está bien establecido quién trata esta patología, pero en la mayoría de hospitales esto no ocurre. Surgen las controversias entre Servicios distintos, e incluso, dentro de un mismo Servicio por hacerse cargo de una patología bastante prevalente (reflujo, escroto vacío...) o interesante por la baja frecuencia (extrofia). En realidad, muchos especialistas pueden tratar muchas patologías sin que nadie lo regule.

- Pérdida de experiencia. Al no existir planificación ni regulación, no se han establecido unos niveles de complejidad-frecuencia, de modo que "cualquier hospital puede hacerse cargo de cualquier patología de UP por compleja e infrecuente que sea". Esto conduce a una minimización y dispersión de las experiencias en patologias que precisamente requieren todo lo contrario.

- Problemas de recursos. Fruto de la enorme dispersión de casos, resulta que los hospitales sin UP pueden tener problemas de dotación adecuada para realizar, por ejemplo, estudios urodinámicos, endourología con pequeños calibres, litotricia, etc. Y los hospitales con UP pueden, también tener problemas para justificar los recursos solicitados dada la dispersión ya citada.

- Falta de conexión hospital infantil-hospital de adultos. Sólo en tres hospitales la UP está dentro de la Urología. Esto tiene la ventaja de la 
continuidad que bastantes procesos requieren (extrofias, válvulas uretrales, vejigas neuropáticas, etc.). Estos niños, cuando se hacen adultos, siguen siendo tratados en el mismo hospital por un Servicio de Urología que conoce su patología, su historia clínica y sus circunstancias personales. Esto es bueno para el niño.

En la mayoría de hospitales, sobre todo infantiles, es difícil la interconexión regular y periódica entre Cirugía Pediátrica o Urología Pediátrica con Urología General. Cuando el niño pasa a adolescente, debe pasar a un Servicio de Urología poco familiarizado con su patología, que desconoce su historia y que se ha de encargar de él para el resto de su vida. Esto no es bueno para nadie.

Formación del residente. Muy pocos residentes de Urología tienen una rotación superior a tres meses por una Unidad de UP. Sin formación no puede haber buena asistencia. El urólogo necesita conocer la UP por diversos motivos:

1. Porque es parte de su especialidad.

2. Porque pueden solucionar patologías muy prevalentes donde no exista UP ni cirugía pediátrica.

3. Porque tendrán que tratar a los niños de UP cuando se hagan adultos.

Los residentes de Cirugía Pediátrica de los hospitales con UP, rotan todos por ella, pero no es así en los hospitales con Cirugía Pediátrica pero sin UP; esta rotación se pierde, se diluye, en la asistencia diaria de todo tipo de patologías de cirugía pediátrica.

El cirujano pediátrico precisa formación específica en UP, por los mismos dos primeros motivos que el urólogo.

\section{PROPUESTA EVOLUTIVA}

Tenemos un diagnóstico de la situación actual de la Urología Pediátrica en España. Del análisis del mismo surgen claras las carencias que de alguna forma deberíamos intentar subsanar.

Llegar a una situación ideal, como toda utopía, es actualmente impracticable pero se puede programar para conseguir en el futuro. Para acercarnos es menester contemplar un corto, un medio y un largo plazo en aspectos docentes y organizativos.

\section{A corto plazo}

Aspectos docentes

El objetivo a corto plazo sería que todos los residentes de Urología y Cirugía Pediátrica pudiesen tener una estancia participativa en una Unidad de Urología Pediátrica, que reuniese las condiciones asistenciales y docentes para que el futuro especialista adquiera esa mínima formación indispensable para el tratamiento de los casos sencillos. En esta primera etapa bastaría con una "facilitación" para que todos los residentes pudieran asistir un mínimo de tres meses a las Unidades actualmente organizadas.

\section{Aspectos organizativos}

Desde los Ministerios de Sanidad y Consumo y de Educación y Ciencia, se ha solicitado al Consejo Nacional de Especialidades Médicas que se proyecten unas áreas de capacitación específicas (ACE), y definen la ACE como:

"El conjunto de conocimientos, habilidades y actitudes añadidos en profundidad y/o extensión, a los recibidos en el periodo de formación como especialistas y desarrollados sobre una parte del contenido de una o más especialidades, en que ya exista interés asistencial y científico suficiente y una dedicación significativa de un grupo de especialistas".

Desde la Asociación Española de Urología, y la Sociedad Española de Cirugía Pediátrica nos ha llegado el encargo de realizar el proyecto ACE en UP, y lo estamos haciendo contemplando el ordenamiento europeo de UP. El objetivo es que tenga una repercusión real, práctica y vinculante.

\section{A medio plazo}

Aspectos docentes

En el medio plazo sería deseable que esas Unidades estuvieran en condiciones de recibir especialistas recién formados, durante seis meses o un año. Estas estancias estarían destinadas a aquellos profesionales interesados en aprender una parte más "profunda" de la Urología Pediátrica, y eventualmente, dedicarse a ella total o parcialmente.

\section{Aspectos organizativos}

Definidas y acreditadas las Unidades de Urologia Pediátrica existentes, hay que determi- 
nar el número adecuado de Unidades de UP y de especialistas, con previsión de la actividad asistencial y el interés social, regulado en función de las necesidades sanitarias del país.

\section{A largo plazo}

Aspectos docentes

La finalidad ulterior es ampliar-regularhomogeneizar los planes de estudio-trabajo en esta parte de la especialidad para que tanto urólogos como cirujanos pediátricos tengan a su alcance las posibilidades, desde su formación de residentes, a cualquier otro momento de su vida profesional, de recibir una instrucción adecuada, adaptada a sus intereses y necesidades. Es frecuente, y lo será más en un futuro, la movilidad laboral de los especialistas. En función de la misma y de otros factores individuales y colectivos, surgen intereses o deseos de realizar una profundización teórico-práctica en Urología Pediátrica.

\section{Aspectos organizativos}

Realizado un "mapa español de Unidades de Urología Pediátrica”, se deberían estructurar unas Unidades de referencias para determinadas patologías y técnicas que, por su escasa prevalencia pero gran trascendencia, justificarían reunir la experiencia nacional y los recursos en dos o tres centros. Igual que sólo cinco Unidades hacen trasplante renal pediátrico, sólo dos o tres deberían atender extrofias vesicales y cloacales, por ejemplo.

\section{RELACIÓN CON OTRAS ESPECIALIDADES}

En todo momento es necesario mejorar y ampliar la relación con los especialistas que tienen implicaciones en UP, como son Cirugía Pediátrica y Urología fundamentalmente pero también, Obstetricia, Pediatría, etc., tanto a nivel general corporativo, como a nivel individual de cada hospital.

Queda finalmente por analizar la imprescindible necesidad de correlacionar las Unidades de Urología Pediátrica con los Servicios de Urología, que son los que deberán atender a todos los pacientes de UP con patología que influyan en su vida adulta.
Así como en la primera infancia es imprescindible contar con Servicios de Pediatría que den a nuestros pacientes soporte asistencial, cada vez es más necesario que Unidades Oncológicas, Uroneurológicas y Reconstructivas, participen en la asistencia y luego se hagan cargo de los pacientes que, superada la edad pediátrica, se transforman en adultos.

La colaboración entre UP y Urología General no sólo es deseable, si no necesaria, imprescindible. Un buen punto de encuentro inicial sería fomentar las Unidades de Urología del adolescente (que existen en otros países de Europa y en algunos hospitales españoles) en las que UP y urólogos de adultos trabajen juntos, con una proyección de futuro conjunta.

En este capítulo de intenciones prima nuestro deseo de difundir aspectos de la especialidad en la seguridad de que hay muchos urólogos a los que les interesaría tener desde una mínima formación a una más amplia.

Pero hay que tener en cuenta los medios con los que contamos y qué podríamos hacer para mejorarlos y ponernos a la altura de los países "punteros".

De acuerdo al análisis y encuesta realizado por el Dr. Mouriquand, podemos asumir que nos encontramos en una situación intermedia en Europa.

Creemos que a través de la Asociación Española de Urología, de la Fundación para la Investigación en Urología y de la Sociedad Española de Cirugía Pediátrica, podríamos influir en la organización sanitaria para poder cumplir estos planes y obtener un reconocimiento para quienes opten por estos complementos formativos.

\section{La Urología Pediátrica en Europa en 2001}

Pierre D.E. Mouriquand, MD; FRCS (Eng)

Professeur d'Urologie Pédiatrique. Hôpital Debrousse. Lyon. France

La Urología Pediátrica es una especialidad nueva, siendo nosotros sólo la tercera generación de especialistas en Occidente. En algún país de la Unión Europea, la Urología Pediátrica está consi- 
derada como una especialidad independiente. En la mayoría, se encuentra comprendida en Servicios de Cirugía Pediátrica o Urología.

A través de una encuesta que realicé en 1999, por encargo de la European Society for Pediatric Urology (ESPU), se vio que el 46\% (10-95\%) de las intervenciones en Urología Pediátrica europeas / Turquía incluida) eran realizadas por cirujanos pediátricos; $20 \%$ por urólogos generales $(0-80 \%)$; $3 \%(0-20 \%)$ por cirujanos generales y $31 \%(0-80 \%)$ por urólogos pediátricos, es decir, por cirujanos estrictamente dedicados a esta especialidad.

Estas cifras son imprecisas pero dan una idea del reparto de influencias en Europa. Las variaciones de un país a otro en número de centros practicando nuestra especialidad, son considerables, yendo de una superabundancia como en Suiza (un centro cada 750.000 habitantes) a una penuria preocupante en Turquía (un centro cada 16 millones de habitantes) con una media europea de un centro cada uno a tres millones.

Después de una veintena de años, la Urología Pediátrica sintió la necesidad de identificarse y esa es la razón por la cual yo tuve el privilegio de co-fundar en 1989 The European Society for Paediatric Urology (ESPU), que se ha desarrollado como una sociedad floreciente. Su nivel científico en sus congresos anuales sucesivos se ha manifestado como de primer orden y puede ser comparado, sin sonrojarse, al de la Sección de Urología Pediátrica del The American Academy of Pediatrics (AAP). El número actual de miembros de la ESPU se sitúa entre 300 y 400.

Urge también como muy importante, definir en cada país programas de educación para que los jóvenes cirujanos o urólogos que quieran, puedan completar su especialidad.

Por otro lado, es fundamental definir cuáles son las necesidades óptimas de cada país para regular el número de especialistas necesarios.

No existe homogeneidad en los programas de formación en Europa, pero siempre dentro de la misma encuesta, surgió un consenso en torno a la situación ideal: dos años de Urología General, dos años de Cirugía Pediátrica y dos años de Urología Pediátrica. No había ningún país que contemplara esta situación formativa.
La necesidad anual de nuevos urólogos pediátricos, varía entre 0,25 a 6 por año, según los países, con una media general europea de 1,5 años.

La ESPU, que ha tomado conciencia de este problema desde hace ya unos cuantos años, hace un gran esfuerzo para que la Urología Pediátrica sea reconocida como una especialidad. Diez países de dieciséis interrogados en 1999, deseaban la autonomía de la Urología Pediátrica, esencialmente para elevar la calidad y especificidad de la formación. Cinco estimaban que no se justificaba esa autonomía, siempre que se lograse que las especialidades "madres" ayudaran a la formación completa en Urología Pediátrica.

La medicina está en una etapa de grandes cambios y la Urología Pediátrica no escapa a esta transformación profunda. En Europa comienza a haber oportunidades para todos y no hay que perderlas. La creación de un ordenamiento europeo de centros especializados de referencia en Urología Pediátrica, permitiría una adecuada circulación y una mejor formación de especialistas. Pero esto, también repercutiría sobre la formación de la enfermería y otros auxiliares y, en definitiva, sobre los niños enfermos de uropatías.

Un paso más sería definir centros capaces de dar la mejor asistencia a determinadas patologías poco frecuentes como por ejemplo la extrofia vesical que, a nuestro entender, deberían ser tratadas en uno o dos centros por país. La media anual de niños nacidos con extrofia es de alrededor de ocho por país y año. Por ejemplo, el Reino Unido ha definido un centro al norte y otro al sur del país.

Hay mucho camino por hacer, aunque nos parezca que, en relativamente pocos años, el trayecto recorrido es entusiasmante.

Dr. C. Miguelez Lago

Servicio de Urología Pediátrica

Hospital Materno Infantil Carlos Haya

Avda. Carlos Haya, s/n

29010 Málaga

(Trabajo recibido el 19 septiembre 2003) 